\title{
Canine Assisted Occupational Therapy: Protocol of a Pilot Randomised Control Trial for Children on the Autism Spectrum
}

\author{
Jessica Hill1 ${ }^{*}$, Jenny Ziviani' ${ }^{1}$, Judy Cawdell-Smith ${ }^{2}$, Carlie Driscoll1 ${ }^{1}$ \\ ${ }^{1}$ School of Health and Rehabilitation Sciences, The University of Queensland, Brisbane, Australia \\ ${ }^{2}$ School of Agriculture and Food Sciences, The University of Queensland, Brisbane, Australia \\ Email: *j.r.hill@uq.net.au
}

How to cite this paper: Hill, J., Ziviani, J., Cawdell-Smith, J. and Driscoll, C. (2019) Canine Assisted Occupational Therapy: Protocol of a Pilot Randomised Control Trial for Children on the Autism Spectrum. Open Journal of Pediatrics, 9, 199-217. https://doi.org/10.4236/ojped.2019.93020

Received: June 9, 2019

Accepted: July 14, 2019

Published: July 17, 2019

Copyright () 2019 by author(s) and Scientific Research Publishing Inc. This work is licensed under the Creative Commons Attribution International License (CC BY 4.0).

http://creativecommons.org/licenses/by/4.0/

\begin{abstract}
Autism spectrum disorder is a neurodevelopmental condition characterised by difficulties with communication, social interaction, and restricted and repetitive behaviours. For children with this disorder, the ability to engage with people and activities, and to participate in everyday occupations is compromised. Animal assisted therapy has been proposed as an intervention modality to facilitate development of an individual's social behaviours such as engagement and attention. In spite of the increasing evidence showing the positive impact animal assisted therapy can have for children on the autism spectrum, there remain no universal standards or formalised guidelines for ethical practice with either the clients or the therapy dogs involved. The proposed pilot randomised control trial aims to determine the impact of canine assisted occupational therapy on the on-task behaviours and overall goal attainment of children on the autism spectrum, as well as consider the impact that being involved within therapy sessions has on the therapy dog.
\end{abstract}

\section{Keywords}

Animal Assisted Therapy, Animal Welfare, Autism Spectrum Disorder, Canine Assisted Therapy, Occupational Therapy, Treatment Protocol

\section{Introduction}

Autism spectrum disorder (ASD) is a neurodevelopmental condition, characterized by difficulties in socialization and communication, and restrictive and repetitive behaviours [1]. Children with ASD can experience difficulty engaging (seeking involvement with people and activities) in the everyday tasks necessary 
for home, school and community participation [2] [3]. When participation opportunities do arise, children on the autism spectrum appear more motivated to engage with objects rather than people, limiting social learning opportunities [2] [3] [4] [5]. Although children with ASD are less inclined to engage with humans, there is now developing evidence that they are able to form close relationships with animals [5] [6].

Drawing on the theory of human-animal interaction (HAI), animal assisted interventions (AAI) is the umbrella term used to describe animal assisted therapy (AAT), animal assisted activities (AAA) and animal assisted education (AAE) [7]. There is evidence to suggest that including an animal into therapy sessions with children on the autism spectrum can facilitate desired social behaviours including: motivation, joint attention and engagement in a task [8]-[13]. This affinity with animals may offer a means of enhancing the delivery of interventions such as occupational therapy, the aim of which is to optimize participation in everyday activities.

Human-animal interaction (HAI) is described as a mutual and dynamic relationship between people and animals, affecting physical and psychological health, in addition to, overall welfare [14] [15]. Welfare has been described as the state of an individual when attempting to cope with the stressors of the environment [15]. In spite of the growing evidence pointing to the benefits of this relationship for human health and well-being, few studies have explored the impact HAI may have on animals. Importantly, there are no universal standards and formal guidelines for ethical practice in this field [14]. In this study protocol, the safe and ethical treatment of animals as well as client outcomes will be addressed to advance the development of practice guidelines. Furthermore, of the available research, none have investigated the welfare of therapy dogs involved in occupational therapy sessions, nor within private practice settings rather than engaged in hospital visits [15].

Although a variety of species are incorporated into AAIs (including, but not limited to: horses, cats, and guinea pigs), dogs continue to be the most commonly involved species. This may be a result of their longstanding domestication history, size, ability to train, and sensitivity to human cues [14]. For this reason, only dogs will be the focus of this intervention protocol.

\subsection{Approaches to Supporting Child Engagement within Occupational Therapy Sessions}

Occupational therapy is one of the most frequently accessed services for families with children on the autism spectrum, second only to speech therapy [16] [17]. Focus areas for occupational therapy often include sensory processing, sensory-motor performance, self-care, social-behaviour performance, participation in play and school-based skills [16] [17] [18].

Difficulty with task engagement has been observed to impact individuals diagnosed with ASD across multiple domains of their daily functioning, such as: self-care, play, leisure and school-based tasks [19]. This often results in indi- 
viduals experiencing difficulty with attending to the task and/or materials, or engaging in undesired, non-task related behaviours [19]. As active involvement is essential for goal attainment, it is crucial that therapy interventions are able to engage children on the autism spectrum with the task and with the therapist [3] [20].

Interventions relying on extrinsic motivators, such as reward systems, to entice children to engage, have limited success in enhancing the duration, frequency and sustainability of behaviour [2]. Activities draw on a child's intrinsic motivation to engage show greater success for children on the autism spectrum [2] [21]. Several strategies support a child's intrinsic motivation and engagement within therapy sessions. Providing the child with a sense of choice within the intervention (for example, allowing the child to assist in developing the activity schedule) promotes a sense of control, facilitating engagement with the therapist and with the task [2] [22] [23]. In addition, providing children with whole tasks with multiple steps has been shown to foster greater engagement when compared to repetition of a single component [22] [23]. Scaffolding and grading activities also provide a "just right challenge" tailoring the task to match the child's strengths and limitations, further enhancing children's motivation to persist in goal-directed activities [22].

Specific strategies have been identified to support the engagement of children on the autism spectrum. Beginning the session by incorporating the child's special interests has been posited to provide a more playful environment in which the child is more likely to engage [22] [23]. Incorporating the use of visuals supports, such as activity schedules and social stories, has been shown to enhance the child's sense of autonomy, whilst reducing the need for adult prompting [2] [19] [24] [25].

\subsection{Canine Assisted Interventions with Children on the Autism Spectrum}

Animal assisted therapy is described as a planned, goal-directed, documented intervention directed by a health professional working within the scope of their field [26]. Over the past decade there has been increasing evidence of the positive impact that canine assisted interventions can have on the social behaviours (behaviours between two or more individuals) of children on the autism spectrum [5] [9] [11] [12] [13] [27]-[32]. Incorporating a dog into the learning environments of children is thought to act as an internal motivator, reducing the child's self-consciousness, increasing attention and awareness of their social environment, as well as, their playfulness [33].

Studies exploring canine assisted therapy specifically have demonstrated improvement in a child's social engagement when interacting with a therapy dog, including an increase in verbal and non-verbal communication and desired social behaviours, as well as a decrease in undesired social behaviours (behaviours preventing social engagement) [11] [28] [29] [30] [31]. What remains unanswered, however, is the effect AAT has on goal attainment within traditional 
therapy modalities for children with autism, such as occupational therapy. As this is an "assisted therapy" further exploration of the efficacy of involving therapy dogs within specific therapeutic processes is necessary to establish this as evidence-based practice [34].

\subsection{Aims and Hypothesis}

The purpose of this paper is to construct and document the treatment protocol to be used in a pilot randomised control trial (RCT) which will explore the impact of including a therapy dog into occupational therapy sessions for children on the autism spectrum when compared to usual care occupational therapy. Development of a protocol is crucial due to the current lack of guidelines and standards of safe and ethical practice of animal assisted therapy as an adjunct to occupational therapy [14].

\section{Aim 1}

To determine if including a therapy dog into occupational therapy sessions can increase the amount of time engaged in on-task behaviour within the session for children with ASD.

\section{Hypothesis 1}

Children on the autism spectrum who participate in canine assisted occupational therapy will spend more time actively attending to a task compared to children receiving usual care occupational therapy.

\section{Aim 2}

To determine if including a therapy dog into occupational therapy sessions can lead to greater goal attainment compared to usual care occupational therapy.

\section{Hypothesis 2}

Children on the autism spectrum who participate in canine assisted occupational therapy will obtain greater improvements in goal performance and satisfaction as measured using the Canadian Occupational Performance Measure (COPM) compared to children receiving usual care occupational therapy.

\section{Aim 3}

To ascertain if participation within canine assisted occupational therapy sessions produces elevated stress levels for the therapy dog as measured by differences in salivary cortisol, alpha-amylase, oxytocin and behavioural observation, when compared with days spent at home.

\section{Hypothesis 3}

Dogs who participate in canine assisted occupational therapy sessions will demonstrate increased stress during therapy sessions when compared to their rest state at home.

\section{Method}

\subsection{Study Design}

A pilot randomised control trial will compare canine assisted occupational therapy and usual care occupational therapy for children on the autism spectrum. 
This will be completed in accordance with the CONSORT 2010 Statement: Update guidelines for reporting parallel group randomised trials (Figure 1) [35].

\section{Eligible children:}

- $\quad$ Aged 4:0 to 6:11 years

- Proficient with English

- Diagnosis of autism spectrum disorder level 1 or 2

- No additional co-diagnosis

- Previous positive experience with dogs

interested, no further contact


Figure 1. CONSORT flow chart of canine assisted occupational therapy [35]. 
There will be two groups involved within this study, the canine assisted occupational therapy intervention group and the control group. Human ethics approval has been provided (Approval number 2018000707) by the University of Queensland Human Research Ethic Committee. Animal ethical clearance has been provided (Approval number SAFS/152/18) by the University of Queensland Production and Companion Animals AEL (PCA) Ethics Committee.

\subsection{Recruitment}

A total of twenty-two children will be recruited between the ages of four years and six years and eleven months. A narrow age range will be included in order to reduce developmental variability. Children will have a diagnosis of autism spectrum disorder level one or two according to the Diagnostic and Statistical Manual of Mental Disorders, 5th edition (DSM-5) [1], confirmed by a paediatrician or child psychiatrist. A letter will be sent to parents connected to Autism Queensland and the Autism Advisory service in Brisbane, Queensland asking for expression of interest. Interested families meeting the inclusion criteria will then be formally invited to participate in the study.

\subsubsection{Inclusion Criteria for Participants}

Children will be eligible to take part within the study if they are between the ages of four years and 6 years and eleven months, are proficient with English, and have a clinical diagnosis of autism spectrum disorder level one or two according to the DSM-5 with no additional diagnosis. The Autism Spectrum Diagnostic Scale-2nd Edition (ADOS-2) [36] will have been completed either by the first author or other trained health professional to ensure consistency of diagnosis. Children must have had a previous positive experience interacting with dogs as measured by The Children's Attitudes and Behaviours towards Animals (CABTA) scale [37]. The CABTA will be completed by parents to ensure that participants have no history of fear or violence towards dogs.

\subsubsection{Exclusion Criteria for Participants}

Participants not meeting the inclusion criteria will be excluded from this study. Other exclusion criteria include: a secondary diagnosis, children and/or parents who are currently immunosuppressed and, current or previous clients of the therapist completing the intervention (first author).

\subsubsection{Expectations of the Therapist/Handler}

The therapist working with her dog within this program will hold a bachelor's degree in occupational therapy and will perform interventions within the scope of the occupational therapy profession. The therapist must have completed additional handler training (minimum five-day, in person, handler-canine training) allowing safe and ethical practice. Topics included within this training program would include: what is AAT, impact of human-animal interaction, client and animal ethics, animal well-being (including zoonosis), documentation and reporting, policies and procedures, insurance, canine body language, interven- 
tions, and current research. The therapy dog will be owned and cared for by the treating therapist. The therapist will take responsibility for correct hygiene and grooming of the dog. The dog will be hydro bathed a minimum of once a fortnight. All therapy equipment will be washed a minimum of once a week or as needed following use in sessions. Informed consent will be obtained by the child's parent prior to the initial session, providing permission for the child to participate in the study and to work with the therapy dog. Easy access to hand sanitiser, disinfectant wipes and cleaning products if needed will be ensured at all times and the therapist will prompt the child to wash his or her hands with hand sanitiser prior to leaving each session. All toys that come into contact with the dog or the child will be washed with disinfectant after the session. In addition, the therapist will abide by all items outlined within the animal code of ethics (Appendix 1).

\subsubsection{Inclusion Criteria for the Dogs within Therapy Sessions}

The dog participating within this program will be over twelve months of age [14] [38] [39]. The dog will have local council registration. The dog will be well socialised to people of all ages, and have completed therapy dog training and assessment for temperament and behaviour, including: friendly, calm and predictable reactions to children, medical equipment (e.g., crutches), client infirmities (e.g., uneven gate, stimming), loud noises, other animals, a range of touches including hugs, items being thrown, toys, and fast movements (e.g., children running); comfortable in a range of settings; emotionally mature; drawn to people, and; confident [14] [15] [26] [39]. Reassessments of the included dog will occur every twelve months unless concerns are noted earlier by the therapist. The dog must complete a bi-annual, full veterinarian health check to ensure physical and emotional health, and confirming vaccinations as well as, have regular treatments for fleas, ticks and parasites [14] [15] [26] [39]. The dog will be able to complete the following tasks and commands consistently under distractions and without food rewards: walk on loose lead through a crowd of people, sit, drop and stay, recall, leave it, on your mat, accept a friendly stranger and sit politely for patting [14] [15] [26] [39]. The dog will act calmly and predictably when working off lead in a contained environment, such as a clinic room [14] [15] [26] [39].

\subsubsection{Exclusion Criteria for the Dogs within Therapy Sessions}

The dog will not be eligible to participate in the therapy program if at any time the inclusion criteria are not met. The dog will also be excluded if the following behaviours are displayed: overly enthusiastic, jumping, mouthing, biting, aloof to people, fearful, aggressive (including resource guarding), or owner focused [14] [38] [39].

\subsection{Randomization}

Randomisation will take place after recruitment. Children will be randomised 
into two groups, canine assisted occupational therapy intervention $(\mathrm{n}=11)$ and a waitlist control group $(\mathrm{n}=11)$ using a centralised electronic allocation system. The waitlist control group will be offered a block of animal assisted occupational therapy directly after the research period.

\subsection{Blinding}

Researcher, child or parent blinding will not be possible throughout the study. Video footage recorded from the therapy sessions to assess child on-task behaviour and therapist fidelity will be coded by two non-study personnel (Honours students at the University of Queensland) who will be blinded to the study aims and hypothesis. This will be done to reduce the possibility of bias amongst the coders. Video footage recorded to assess canine behaviour within the therapy sessions will be coded by two additional non-study personnel (animal assisted psychologist and an animal assisted occupational therapist) one of whom is blinded to the study aims and hypothesis.

\subsection{Data Collection}

All therapist and child interactions during the therapy session will be video recorded and a total of 44 (first and last session of all children) will be coded by two non-study personnel for the time spent actively attending to the therapy task, as well as, fidelity of therapist delivered intervention. On-task behaviour is defined as "physical contact with one or more objects in a manner that could result in completion of a task" [40: p. 262]. Examples of on-task behaviours within this study may include: collecting materials needed to complete the task, manipulating materials in a way that is directed towards task completion and requesting assistance from the therapist [40].

To assess goal attainment, the Canadian Occupational Performance Measure (COPM) will be completed pre-and post-intervention by the child's parent. The COPM is a well-researched, widely used tool within paediatric rehabilitation to assist with goal setting, as well as, outcome measurement [41] [42]. It provides therapists with an understanding of the client's performance and satisfaction in self-care, leisure and productivity [41] [42]. Although initially designed to be completed with the client directly, this is not possible with children under the age of eight who experience difficulty with self-assessment [41]. Because of this, parents must act as a proxy, reliably identifying meaningful goals for their child [42]. The COPM will be completed in the initial parent session with the therapist, as well as, the final intervention session with the therapist and parent.

In order to assess the wellbeing of the therapy dogs involved within this study, salivary samples will be taken at multiple intervals to assess for changes in cortisol, alpha amylase, and oxytocin levels. The adrenocortical hormone cortisol has been the most commonly referenced biomarker when assessing psychological arousal and is regularly used as a biomarker for animal welfare [15]. An increase in cortisol will occur as a result of the activation of the hypothalamic-pituitary-adrenal 
axis in response to a stressful stimulus [15]. Although acknowledged as a useful biomarker of animal welfare, several limitations have been identified [15]. Firstly, cortisol levels within dogs are characterised by high intra- and inter-variability, and are impacted by several demographic and environmental factors, therefore, limiting its generalisability [15]. In addition, when using cortisol as a biomarker to assess animal welfare, it should be understood that elevated levels of cortisol can reflect excitement [15]. Because of these limitations, alpha amylase (sAA) will also be assessed. Alpha amylase is a protein released by the parotid gland as a result of psychological or physical stress [43]. During periods of stress an increased level of sAA is released into saliva [43]. Saliva samples to assess sAA will be taken at the same time intervals as the cortisol [43].

Oxytocin is a neuropeptide related to social behaviour, cognition and stress responses in mammals [44]. Oxytocin is often referred to as the "bonding" or "love-hormone" and has been used as a biomarker to assess HAI. It has also recently been posited as a potential biomarker for animal welfare and, therefore, will be the third salivary measure assessed within our study [44] [45].

Experimental samples will be collected from the dog at multiple intervals during the treatment period using SalivaBio Children's Swabs [44] [46] (Table 1; Table 2). No food will be provided to the dog within 60 minutes prior to the Table 1. Canine sample collection schedule.

\begin{tabular}{|c|c|c|c|}
\hline Baseline sample & & Treatment sample & \\
\hline \multirow[t]{14}{*}{ 7:00 am - 5:00 pm } & Typical day at home & 7:00 am - 7:55 am & Arrival at clinic-Rest \\
\hline & & 7:55 am - 8:00 am & Pre-therapy sample taken \\
\hline & & 8:00 am - 9:00 am & $\begin{array}{l}\text { Client } 1 \text { (sample taken } 20 \text { mins } \\
\text { after active interaction with child) }\end{array}$ \\
\hline & & 9:00 am - 9:30 am & Rest \\
\hline & & $9: 30$ am - 9:35 am & Post-therapy sample taken \\
\hline & & $9: 35$ am - 10:35 am & Client 2 (no sample taken) \\
\hline & & $10: 35 \mathrm{am}-11: 00 \mathrm{am}$ & Rest \\
\hline & & $11: 00 \mathrm{am}-12: 00 \mathrm{pm}$ & Client 3 (no sample taken) \\
\hline & & $12: 00 \mathrm{pm}-12: 30 \mathrm{pm}$ & Rest \\
\hline & & $12: 30 \mathrm{pm}-1: 30 \mathrm{pm}$ & Client 4 (no sample taken) \\
\hline & & $1: 30 \mathrm{pm}-2: 00 \mathrm{pm}$ & Rest \\
\hline & & $2: 00 \mathrm{pm}-3: 00 \mathrm{pm}$ & Client 5 (no sample taken) \\
\hline & & $3: 00 \mathrm{pm}-3: 25 \mathrm{pm}$ & Rest \\
\hline & & $3: 25 \mathrm{pm}-3: 30 \mathrm{pm}$ & Pre-therapy sample taken \\
\hline $5: 00 \mathrm{pm}-5: 30 \mathrm{pm}$ & Handler arrives home & $3: 30 \mathrm{pm}-4: 30 \mathrm{pm}$ & $\begin{array}{l}\text { Client } 6 \text { (sample taken } 20 \text { mins } \\
\text { after active interaction with child) }\end{array}$ \\
\hline $5: 30 \mathrm{pm}-6: 00 \mathrm{pm}$ & Rest & $4: 30 \mathrm{pm}-4: 55 \mathrm{pm}$ & Rest \\
\hline $6: 00 \mathrm{pm}-6: 05 \mathrm{pm}$ & Baseline sample taken & 4:55 pm - 5:00 pm & Post-therapy sample taken \\
\hline
\end{tabular}


Table 2. Canine sample collection schedule.

\begin{tabular}{cccc}
\hline Week & Day & Session & Number of samples \\
\hline 1 & Wednesday & Baseline & 1 \\
1 & Thursday & First & 3 \\
1 & Thursday & Last & 3 \\
4 & Wednesday & Baseline & 1 \\
4 & Thursday & First & 3 \\
4 & Thursday & Last & 3 \\
7 & Wednesday & Baseline & 1 \\
7 & Thursday & First & 3 \\
7 & Thursday & Last & 3 \\
\hline
\end{tabular}

sample being taken to prevent contamination of the sample [44] [47]. The swab will be placed between the dog's mandibular teeth and cheek by the first author for a total of 90 seconds, whilst gently holding her mouth shut to prevent swallowing [44] [47]. A treat will be given to the dog after the final sample has been taken [44] [47]. Samples will be stored within a sample tube and frozen at $-40^{\circ} \mathrm{C}$ immediately after extraction and transported on ice to be frozen at $-80^{\circ} \mathrm{C}$ within 24 hours after initial collection [47].

In order to increase the robustness of a study, evaluation of biomarkers such as cortisol and oxytocin levels is often paired with observation of stress-associated behaviour [39]. Behaviour occurs as a result of the dog's physical and mental state, with abnormal behaviour being a sign of stress or pain [39]. Observational checklists can be an effective method of assessing animal welfare as they are able to provide detailed information on the animals in a non-invasive manner [39]. Therefore, The Pet Assisted Therapy-Welfare Assessment Tool (PAT-WAT) will be completed [39]. Dog behaviour will be video recorded and coded by two trained, non-study personnel during weeks one, four and seven, during the first and final session of a treating day.

After completion of the treatment period, the parents of the canine assisted occupational therapy treatment group will be contacted and invited to participate in an informal phone interview. Furthermore, after the control group have completed their canine assisted occupational therapy treatment period, they too will be contacted and invited to participate in the informal phone interview. Follow up sessions will be conducted as semi-structured, in-depth interviews, in an attempt to gain an understanding of the parent's experience of their child participating in animal assisted occupational therapy [48].

A treatment manual outlining session structure and implementation of the canine assisted intervention and usual care occupational therapy has been developed and is outlined in Table 3. All therapy sessions will be digitally video-recorded and a total of 22 (one video from each child selected at random) will be coded by independent coders for therapist fidelity using a checklist developed for this study. 
Table 3. Therapy procedure for canine assisted occupational therapy and usual care.

\begin{tabular}{|c|c|c|}
\hline Session outlines & Canine assisted occupational therapy & Usual care \\
\hline $\begin{array}{l}\text { Initial parent } \\
\text { information session } \\
\text { (1) }\end{array}$ & $\begin{array}{l}\text { - Goal setting session with the parent and therapy dog } \\
\text { present. Completion of the COPM. } \\
\text { - Child strengths and interests identified through discussion } \\
\text { with the parents. } \\
\text { - Discussion of home practice expectations. }\end{array}$ & $\begin{array}{l}\text { - Goal setting session with the parent. Completion of } \\
\text { COPM. } \\
\text { - Child strengths and interests identified through } \\
\text { discussion with the parents. } \\
\text { - Discussion of home practice expectations. }\end{array}$ \\
\hline $\begin{array}{l}\text { Initial session with } \\
\text { the child ( } 2 \text { ) }\end{array}$ & $\begin{array}{l}\text { - Therapist to introduce themselves to the child. } \\
\text { - Introduce child to therapy dog providing child with photo } \\
\text { of the dog with dog's likes written on the back. } \\
\text { - Child to draw a picture of self and interests for the therapy } \\
\text { dog allowing occupational therapist to make behavioural } \\
\text { observations including following instructions and } \\
\text { attention. } \\
\text { - Goal/s will be discussed with child in developmentally } \\
\text { appropriate manner. } \\
\text { - Clinical observations are to be made within the initial } \\
\text { sessions regarding the child's identified goal/s. }\end{array}$ & $\begin{array}{l}\text { - Therapist to introduce themselves to the child. } \\
\text { - Child to draw a picture of self and interests for the } \\
\text { therapist allowing for behavioural observations including } \\
\text { following instructions and attention. } \\
\text { - Goal/s will be discussed with child in developmentally } \\
\text { appropriate manner. } \\
\text { - Clinical observations are to be made within the initial } \\
\text { sessions regarding the child's identified goal/s. } \\
\text { - Session will conclude with the client choosing a sticker } \\
\text { and discussing home practice to show the occupational } \\
\text { therapist next session. }\end{array}$ \\
\hline
\end{tabular}

\section{Intervention}

sessions (3-8)

Follow-up session

(9)

Home program
- Session will conclude with giving the dog his/her "reward treat" and discussing home practice to show the therapy dog next session.

- Entering each session: Discussion of previous week home practice.

- Beginning intervention: The child will be presented with activity visuals planned for the session. Child and therapy dog (if requested) will be involved in choosing activities for session.

- Intervention activities: Interventions performed throughout the session will be "whole tasks" with a meaningful end goal directed towards the child's overall goal/s. The "just right challenge" will be provided within all tasks through thorough task analysis completed by the occupational therapist. In addition to visual supports, other evidence-based behaviour management strategies, such as positive reinforcement of the child's attempts and desired choices will be used throughout the session. Positive reinforcement will include both active and passive involvement of therapy dog. The therapy dog will be actively involved in a minimum of one-therapy activities (including but not limited to: dog pretend play, e.g. puppy tea party; development of social stories involving the dog; self-care skill development, e.g. dressing using dress ups with the dog, board games with the dog as another player). The therapy dog will be passively involved in all other activities (including but not limited to: showing the therapy dog work completed by the child; talking about the therapy dog's emotions; dog themed fine motor tasks, e.g. dog drawing/colouring).

- Ending the session: Sessions will conclude with giving the dog his/her "reward treat" and discussing home practice.

- Session will be completed as initial session without the child present.

- COPM will again be completed by the parent.

One to two functional tasks tailored to child's goal is provided at the end of sessions 2-7.
- Session will be completed as initial session without the child present.

- COPM will again be completed by the parent.

One to two functional tasks tailored to child's goal is provided at the end of sessions 2-7. 
Coding checklists (12 items for the control and 17 items for the treatment group) and will be scored with "full adherence", "partial adherence" or "no adherence" dependent on the therapists' adherence to the treatment manual.

\subsection{Data Analysis}

Analysis will follow standard principles for RCTs, with a focus on comparisons between the intervention and control arm. Analysis will be undertaken on an intention-to-treat basis with statistical significance set at $\mathrm{p}<0.05$. The primary outcome, COPM post intervention phase, will be compared between groups using Mann-Whitney U-test because of the potentially small sample size and possibility of violation of assumptions for inferential statistics. A final decision will be made in consultation with the School statistical advisor. Excel spread sheets and the statistical software SPSS will be used to support data description and analysis. Data collected through parent interviews will be analysed using thematic analysis. Thematic analysis allows researchers to identify and analyse patterns within their data in order to determine which themes are important and present meaning within their study [49].

Analysis of the salivary biomarkers will be completed by expert academics in the University of Queensland Anderson Lab (animal endocrinology laboratory). Microsoft Excel and SPSS software will also be used for the analysis of canine behavioural outcomes.

\section{Study Procedure}

\subsection{Canine Assisted Occupational Therapy Intervention Group}

The canine assisted occupational therapy intervention group will consist of a total of nine, weekly, one-hour sessions. This will include two parent sessions (first and last session) and seven child intervention sessions. The number of sessions and content draws on four years of clinical experience as no published protocols exist. All sessions will take place within a therapy clinic, with an occupational therapist trained in animal assisted therapy (first author), as well as, her therapy dog. An in-depth manual outlining the structure of each session will assist with consistency between sessions, whilst allowing the therapist to tailor interventions for individual clients (see Table 3 ).

All nine sessions will be video, and audio recorded to assess for on-task behaviour, as well as, intervention fidelity. Two cameras will be used within each session positioned at two separate locations within the room. Audio recording of the therapist, in particular, verbal prompts provided to the child, will be captured using a digital voice recorder with an external microphone positioned at a third location within the room.

In the initial session, the Canadian Occupational Performance Measure (COPM) [50] will be completed with the child's parent to determine occupational goal priorities. The COPM will again be completed with the parent during the final session to be used as an outcome measure for goal progression and attainment 
across the nine-week period [50].

Child sessions will consist of evidence-based, goal-directed occupational therapy interventions for children on the autism spectrum [16] [51]. These will be completed by actively involving the therapy dog. Children will also be involved in the co-development of the activity schedule, providing a sense of ownership and control [2] [22] [23]. These schedules will be presented as visuals allowing the child to easily predict what will occur during the session [2] [19] [24] [25]. Each intervention activity will be performed as a "whole task" with a meaningful end goal [22] [23]. Through task analysis, each activity will be graded to provide the "just right challenge" [22]. In addition, the child's interest may also be incorporated into the sessions [22] [23].

\subsection{Usual Care Control Group}

The usual care control group will follow a similar structure to the treatment group (Table 3). A total of nine, weekly, one-hour sessions will be completed by the first author without her therapy dog present. Sessions will be conducted within a therapy clinic. An in-depth manual will be developed allowing for replication (Table 3). Within the initial and final sessions, the COPM [50] will be also completed.

Child sessions will involve evidence-based interventions without the inclusion of the dog. Strategies used to encourage intrinsic motivation to engage will be as indicated within the treatment group with the exclusion of the therapy dog [2] [22] [23].

At the completion of the trial, participants within the usual care control group will be offered a block of eight animal assisted occupational therapy sessions. This will be offered to ensure equity amongst participants and no data will be recorded during this period.

\section{Discussion}

Canine assisted therapy has been proposed to have a positive impact on the social behaviours of children on the autism spectrum such as engagement and attention. However, limited research has been completed to explore the possible impact this may have on goal attainment within traditional therapy modalities such as occupational therapy. Also missing from the literature is an understanding of the impact of canine assisted therapy sessions on the health and well-being of the therapy dog involved, with no current standards or ethical guidelines having been developed. This study has the potential to establish a new means of enhancing the delivery of interventions for children on the autism spectrum such as occupational therapy, whilst also ensuring the safe and ethical treatment of clients and therapy dogs.

\section{Conflicts of Interest}

The authors declare no conflicts of interest regarding the publication of this paper. 


\section{References}

[1] American Psychiatric Association (2013) Diagnositic and Statistical Manual of Mental Disorders: DSM-5. American Psychiatric Association, Washington DC. https://doi.org/10.1176/appi.books.9780890425596

[2] Dearden, J., Emerson, A., Lewis, T. and Papp, R. (2016) Transforming Engagement: A Case Study of Building Intrinsic Motivation in a Child with Autism. British Journal of Sociology of Education, 44, 8-25. https://doi.org/10.1111/1467-8578.12137

[3] Llambias, C., Magill-Evans, J., Smith, V. and Warren, S. (2016) Equine-Assisted Occupational Therapy: Increasing Engagement for Children with Autism Spectrum Disorder. American Journal of Occupational Therapy, 70,

7006220040p1-7006220040p9. https://doi.org/10.5014/ajot.2016.020701

[4] Dawson, G., Toth, K., Abbott, R., Osterling, J., Munson, J., Estes, A. and Liaw, J. (2004) Early Social Attention Impairments in Autism: Social Orienting, Joint Attention, and Attention to Distress. Developmental Psychology, 40, 271-283. https://doi.org/10.1037/0012-1649.40.2.271

[5] Prothmann, A., Ettricht, C. and Prothmann, S. (2009) Preference for, and Responsiveness to, People, Dogs and Objects in Children with Autism. Anthrozoös, 22, 161-171. https://doi.org/10.2752/175303709X434185

[6] Grandin, T., Fine, A.H., O’Haire, M.E., Carlisle, G. and Bowers, C.M. (2015) The Roles of Animals for Individuals with Autism Spectrum Disorder. In: Fine, A.H., Ed., Handbook on Animal-Assisted Therapy. Foundations and Guidelines for Animal-Assisted Interventions, 4th Edition, Elsevier Academic Press, San Diego, 225-236. https://doi.org/10.1016/B978-0-12-801292-5.00016-X

[7] O’Haire, M.E. (2013) Animal-Assisted Intervention for Autism Spectrum Disorder: A Systematic Literature Review. Journal of Autism and Developmental Disorders, 43, 1606-1622. https://doi.org/10.1007/s10803-012-1707-5

[8] Fung (2015) Increasing the Social Communication of a Boy with Autism Using Animal-Assisted Play Therapy: A Case Report. Advances in Mind Body Medicine, 29, 27-31.

[9] Mey, S.C. (2017) Animal Assisted Therapy for Children with Autism. International Journal of Child Developmental and Mental Health, 5, 29-42.

[10] Obrusnikova, I., Bibik, J.M., Cavalier, A.R. and Kyle, M. (2012) Integrating Therapy Dog Teams in a Physical Activity Program for Children with Autism Spectrum Disorders. Journal of Physical Education, Recreation \& Dance, 83, 37-48. https://doi.org/10.1080/07303084.2012.10598794

[11] Petrongelli-Halloran, L.M. (2010) Evaluation of Prosocial Behaviors during Animal-Assisted Therapy for Children with Pervasive Developmental Disorders. Adler School of Professional Psychology, Chicago.

[12] Solomon, O. (2010) What a Dog Can Do: Children with Autism and Therapy Dogs in Social Interaction. Ethos, 38, 143-166. https://doi.org/10.1111/j.1548-1352.2010.01085.x

[13] Stevenson, K., Jarred, S., Hinchcliffe, V. and Roberts, K. (2015) Can a Dog Be Used as a Motivator to Develop Social Interaction and Engagement with Teachers for Students with Autism? Support for Learning, 30, 341-363.

https://doi.org/10.1111/1467-9604.12105

[14] Gee, N.R., Hurley, K.J. and Rawlings, J.M. (2016) From the Dog's Perspective: Welfare Implications of HAI Research and Practice. In: Freund, L.S., McCune, S., Esposito, L., Gee, N.R. and McCardle, P., Eds., The Social Neuroscience of Human-Animal 
Interaction, American Psychological Association, Washington DC, 217-237. https://doi.org/10.1037/14856-013

[15] Glenk, L.M. (2017) Current Perspectives on the Therapy Dog Welfare in Animal-Assisted Interventions. Animals, 7, pii: E7. https://doi.org/10.3390/ani7020007

[16] Ashburner, J., Rodger, S., Ziviani, J. and Jones, J. (2014) Occuptional Therapy Services for People with Autism Spectrum Disorders: Current State of Play, Use of Evidence and Future Learning Priorities. Australian Occupational Therapy Journal, 61, 110-120. https://doi.org/10.1111/1440-1630.12083

[17] Bagatell, N. and Mason, A.E. (2015) Looking Backward, Thinking Forward: Occupaional Therapy and Autism Spectrum Disorders. OTJR: Occupation, Participation and Health, 35, 34-41. https://doi.org/10.1177/1539449214557795

[18] Case-Smith, J. and Arbesman, M. (2008) Evidence-Based Review of Interventions for Autism Used in or of Relevance to Occupational Therapy. The American Journal of Occupational Therapy, 62, 416-429. https://doi.org/10.5014/ajot.62.4.416

[19] Palmen, A. and Didden, R. (2012) Task Engagement in Young Adults with High-Functioning Autism Spectrum Disorders: Generalization Effects of Behavioural Skills Training. Research in Autism Spectrum Disorders, 6, 1377-1388. https://doi.org/10.1016/j.rasd.2012.05.010

[20] King, G., Currie, M. and Petersen, P. (2014) Review: Child and Parent Engagement in the Mental Health Intervention Process: A Motivational Framework. Child and Adolescent Mental Health, 19, 2-8. https://doi.org/10.1111/camh.12015

[21] Sams, M.J., Fortney, E.V. and Willenbring, S. (2006) Occupational Therapy Incorporating Animals for Children with Autism: A Pilot Investigation. American Journal of Occupational Therapy, 60, 268-274. https://doi.org/10.5014/ajot.60.3.268

[22] Case-Smith, J. (2010) An Overview of Occupational Therapy for Children. In: Case-Smith and O'Brian, Ed., Occupational Therapy for Children, 6th Edition, Elsevier, Amsterdam, 5-8.

[23] Koegel, L.K., Singh, A.K. and Koegel, R.L. (2010) Improving Motivation for Academics in Children with Autism. Journal of Autism \& Developmental Disorders, 40, 1057-1066. https://doi.org/10.1007/s10803-010-0962-6

[24] Bryan, L.C. and Gast, D.L. (2000) Teaching On-Task and On-Schedule Behaviours to High-Functioning Children with Autism via Picture Activity Schedules. Journal of Autism \& Developmental Disorders, 30, 553-567. https://doi.org/10.1023/A:1005687310346

[25] Massey, G.N. and Wheeler, J.J. (2000) Acquisition and Generalization of Activity Schedules and Their Effects on Task Engagement in a Young Child with Autism in an Inclusive Pre-School Classroom. Education and Training in Mental Retardation and Developmental Disabilities, 35, 326-335.

[26] International Association of Human Animal Interaction Organisations (2015) The IAHAIO Definitions for Animal-Assisted Intervention and Guidelines for Wellness of Animals Involved. In: Fine, A., Ed., Handbook on Animal Assisted Therapy. Foundations and Guidelines of Animal-Assisted Interventions, 4th Edition, Elsevier, Amsterdam, 414-418.

[27] Fung and Leung (2014) Pilot Study Investigating the Role of Therapy Dogs in Facilitating Social Interaction among Children with Autism. Journal of Contemporary Psychotherapy, 44, 253-262. https://doi.org/10.1007/s10879-014-9274-z

[28] Grigore, A.A. and Rusu, A.S. (2014) Interaction with a Therapy Dog Enhances the Effects of Social Story Method in Autistic Children. Society \& Animals. Journal of Human-Animal Studies, 22, 241-261. https://doi.org/10.1163/15685306-12341326 
[29] Martin, F. and Farnum, J. (2002) Animal-Assisted Therapy for Children with Pervasive Developmental Disorders. West Journal of Nursing Research, 24, 657-670. https://doi.org/10.1177/019394502320555403

[30] Redefer, L.A. and Goodman, J.F. (1989) Brief Report: Pet-Facilitated Therapy with Autistic Children. Journal of Autism and Developmental Disorders, 19, 461-467. https://doi.org/10.1007/BF02212943

[31] Silva, K., Correia, R., Lima, M., Magalhães, A. and de Sousa, L. (2011) Can Dogs Prime Autistic Children for Therapy? Evidence from a Single Case Study. Journal of Alternative and Complementary Medicine, 17, 655-659. https://doi.org/10.1089/acm.2010.0436

[32] Welsh, K.C. (2010) The Use of Dogs to Impact Joint Attention in Children with Autism Spectrum Disorders.

[33] Hart, L.A. and Yamamoto, M. (2015) Recruiting Psychosocial Health Effects of Animals for Families and Communities: Transition to Practice. In: Fine, A., Ed., Handbook on Animal Assisted Therapy. Foundations and Guidelines of Animal-Assisted Interventions, 4th Edition, Elsevier, Amsterdam, 60. https://doi.org/10.1016/B978-0-12-801292-5.00006-7

[34] Fine, T.P. and Elvove, E. (2015) Forward Thinking: The Evolving Field of Human-Animal Interactions. In: Fine, A.H., Ed., Handbook of Animal Assisted Therapy: Foundations and Guidelines for Animal-Assisted Interventions, 4th Edition, Elsevier, Amsterdam, 33. https://doi.org/10.1016/B978-0-12-801292-5.00003-1

[35] Schulz, K., Altman, D. and Moher, D. (2010) CONSORT 2010 Statement: Update Guidelines for Reporting Parallel Group Randomised Trials. BMC Medicine, 8, 18. https://doi.org/10.1186/1741-7015-8-18

[36] Lord, C., Rutter, M., DiLavore, P., Risi, S., Gotham, K. and Bishop, S. (2012) Autism Diagnostic Observation Schedule, Second Edition (ADOS-2), Modules 2 \& 3. Western Psychological Services, USA.

[37] Guymer, E., Mellor, D., Luk, E. and Pearse, V. (2001) The Development of a Screening Questionnaire for Childhood Cruelty to Animals. Journal of Child Psychology and Psychiatry, 42, 1057-1063. https://doi.org/10.1111/1469-7610.00805

[38] Mongillo, P., Pitteri, E., Adamelli, S., Bonichini, S., Farina, L. and Marinelli, L. (2015) Validation of a Selection Protocol of Dogs Involved in Animal-Assisted Intervention. Journal of Veterinary Behavior. Clinical Applications and Research, 10, 103-110. https://doi.org/10.1016/j.jveb.2014.11.005

[39] Ng, Z., Albright, J., Fine, A.H. and Peralta, J. (2015) Our Ethical and Moral Resonsibility: Ensuring the Welfare of Therapy Animals. In: Handbook on Animal-Assisted Therapy: Foundations and Guidelines for Animal-Assisted Interventions, Elsevier, Amsterdam, 357-366. https://doi.org/10.1016/B978-0-12-801292-5.00026-2

[40] Tasky, K.K., Rudrud, E.H., Schulze, K.A. and Rapp, J.T. (2008) Using Choice to Increase On-Task Behavior in Individuals with Traumatic Brain Injury. Journal of Applied Behaviour Analysis, 41, 261-265. https://doi.org/10.1901/jaba.2008.41-261

[41] Cusick, A., Lannin, N.A. and Lowe, K. (2007) Adapting the Canadian Occupational Performance Measure for Use in a Paediatric Clinical Trial. Disability and Rehabilitation, 29, 761-766. https://doi.org/10.1080/09638280600929201

[42] Verkerk, G., Wolf, M.J., Louwers, A.M., Meester-Delver, A. and Nollet, F. (2005) The Reproducibility and Validity of the Canadian Occupational Performance Measure in Parents of Children with Disabities. Journal of Clinical Rehabilitation, 20, 980-988. https://doi.org/10.1177/0269215506070703 
[43] Krause-Parello, C.A. and Friedmann, E. (2014) The Effects of an Animal Assisted Intervention on Salivary Alpha-Amylase, Salivary Immunoglobulin and Heart Rate during Forensic Interviews in Child Sexual Abuse Cases. Anthrozoös, 27, 581-590. https://doi.org/10.2752/089279314X14072268688005

[44] MacLean, E., Gesquiere, L., Gee, N.R., Levy, K., Martin, W. and Carter, C. (2018) Validation of Salivary Oxytocin and Vasopressing as Biomarkers in Domestic Dogs. Journal of Neuroscience Methods, 283, 67-76. https://doi.org/10.1016/j.jneumeth.2017.08.033

[45] McCune, S., Kruger, K., Griffin, J.A., Esposito, L., Freund, L.S., Bures, R., Hurley, K.J. and Gee, N.R. (2015) Strengthening the Foundations of Human-Animal Interaction Research: Recent Developments in Rapidly-Growing Field. In: Fine, A., Ed., Handbook on Animal-Assisted Therapy: Foundations and Guidelines for Animal-Assisted Interventions, Elsevier, Amsterdam, 411.

[46] Salimetrics (2019) How to Collect Saliva: Collection Methods and Devices. https://salimetrics.com/saliva-collection-handbook/\#saliva-collection-methods

[47] Torres, S.M., Furrow, E., Souze, C.P., Granick, J.L., De Jong, E.P., Griffin, T.J. and Wang, X. (2018) Salivary Proteomics of Healthy Dogs: An in Depth Catalog. PLoS ONE, 13, e0191307. https://doi.org/10.1371/journal.pone.0191307

[48] Groenwald, T. (2004) A Phenomenological Research Design Illustrated. International Journal of Qualitative Methods, 3, 1-26. https://doi.org/10.1177/160940690400300104

[49] Joffe, H. (2011) Thematic Analysis. In: Harper, D. and Thompson, A.R., Eds., Qualitative Research Methods in Mental Health and Psychotherapy: A Guide for Students and Practitioners, John Wiley \& Sons, Ltd., Hoboken, 209. https://doi.org/10.1002/9781119973249.ch15

[50] Law, M., Baptiste, S., McColl, M., Opzoomer, A., Polatajko, H. and Pollock, N. (1990) The Canadian Occupational Performance Measure: An Outcome Measure for Occupational Therapy. The Canadian Journal of Occupational Therapy, 57, 82-87. https://doi.org/10.1177/000841749005700207

[51] Tomchek, S., LaVesser, P. and Watling, R. (2015) Scope of Occupational Therapy Services for Individuals with Autism Spectrum Disorder across the Life Course. The American Journal of Occupational Therapy, 69, 1-12. https://doi.org/10.5014/ajot.2015.696S18

[52] Ward, E. (2017) Zoonotic Diseases in Dogs. VCA. https://vcahospitals.com/know-your-pet/zoonotic-diseases-in-dogs

[53] Queensland Government (2017) Animal Care Protection Act 2001. https://www.legislation.qld.gov.au/view/whole/html/inforce/current/act-2001-064 


\section{Appendix 1. Animal Welfare and Code of Ethics}

\section{Animal Welfare and Code of Ethics}

The term "animal welfare" is defined as the "state of the animal as it attempts to cope with its environment" [14: p. 218]. The five freedoms (1969) have frequently been referenced within HAI literature, to conceptualise animal welfare [14] [39]. These "five freedoms" included: 1) freedom from hunger and thirst, 2) freedom from discomfort, 3) freedom from pain and injury, or disease, 4) freedom to express normal behaviour, 5) freedom from fear and distress [14] [39]. From these, a broader characterisation was developed to include three main concepts: physical, affective and nature [39]. From these three concepts our protocol was developed.

\section{Physical}

To ensure the health and well-being of the dogs involved, all dogs incorporated into therapy sessions will receive full veterinary health checks bi-yearly to explore possible physical injuries or zoonosis [26] [52]. A faecal test will be administered at the veterinarian's discretion [39]. Dogs involved in therapy will be up to date with all medical treatments such as vaccinations and parasite protection at the frequency required, as directed by a veterinarian [26] [39]. The health and well-being of the therapy dog is the responsibility of the therapist and, therefore, will be under their constant supervision [39]. If a situation is deemed by the therapist to be "unsafe" or "distressing" to the therapy dog it is their responsibility to therapeutically intervene, ending the interaction [39]. If it is observed that the dog may be injured or unwell, the dog's involvement within the session will be ended immediately and appropriate veterinary care will be sought if necessary [39]. Quantity of treats provided to the therapy dog will be monitored by the therapist with recommendations provided by a veterinarian based on breed, size, and weight [39].

\section{Affective}

The therapist will ensure that the transport of the dog to and from the clinic is safe and comfortable [39]. The dog must not display any form of car sickness. The dog will be worked off lead at all times or on loose flat collar if required (e.g., if outside) allowing the dog the opportunity to move away if uncomfortable [14] [39]. The dog is to never be "held" in an interaction with a client, by the therapist, instead the therapy dog will choose to interact on their own accord [14] [39]. The therapist will ensure that a clean mat and bowl of water is placed safely in the room (away from places the water can easily be spilt) [26]. The mat is to be used as a "safe zone" if the dog appears uncomfortable and is needing space. The client is not allowed to physically enter this space during this time.

\section{Nature}

The therapist is to ensure the dogs are provided with a break every one-to-two hours depending on their previous workload. This break is to be a minimum of ten minutes outside allowing the dog to sniff, play, urinate and defecate if needed. Dogs are to work with a maximum of six clients per day. Dogs will not 
be made to work consecutive days and will be provided with a minimum of one day rest between working days. Additional rest days are to be provided if the dog shows signs of stress or fatigue. The dog's environment is monitored to ensure it is comfortable for the dog (e.g., temperature, clutter).

In addition to the above, all therapists following this treatment protocol will ensure they meet their legal obligations under the Animal Care and Protection Act 2001 and Australian Code for the Care and use of Animals for Scientific Purposes 2013 [53]. 